\title{
Changes in Femoral Stem Geometry Reduce Intraoperative Femoral Fracture Rates in Total Hip Replacement
}

\author{
Tatsuya Sueyoshi, Michael E. Berend, John B. Meding, Robert A. Malinzak, \\ Wesley G. Lackey, Merrill A. Ritter \\ Center for Hip and Knee Surgery, Joint Replacement Surgeons of Indiana Research Foundation (JRSI), \\ Mooresville, IN, USA \\ Email: jrsiresearch1@gmail.com
}

Received 20 March 2015; accepted 6 May 2015; published 8 May 2015

Copyright (C) 2015 by authors and Scientific Research Publishing Inc.

This work is licensed under the Creative Commons Attribution International License (CC BY).

http://creativecommons.org/licenses/by/4.0/

c) (i) Open Access

\begin{abstract}
Intraoperative fracture rates in total hip arthroplasty (THA) have been associated with uncemented stems, anterolateral surgical approach, diagnoses other than osteoarthritis, and female gender. The purpose of this study was to investigate if changes in stem design would influence intraoperative fracture rates. 1497 THA with Bi-Metric stems were compared to 1277 THA with Echo Bi-Metric Full Proximal Profile stems and 366 THA with Echo Bi-Metric Reduced Proximal Profile stems. The intraoperative fractures rates were $5.48 \%, 1.41 \%$ and $1.37 \%$ for Bi-Metric and Echo Bi-Metric with standard and reduced proximal geometry stems, respectively. Stem survivorship with aseptic loosening as an endpoint was $100 \%$ in all cohorts at the final follow-up. We conclude that changes in femoral stem geometry in uncemented THA reduce intraoperative fracture rates while preserving fixation.
\end{abstract}

\section{Keywords}

Total Hip Arthroplasty, Stem Design, Intraoperative Fractures, Survivorship

\section{Introduction}

Intraoperative fracture rates in total hip arthroplasty (THA) have been associated with uncemented stems, anterolateral surgical approach, and female gender [1]. We used uncemented, porous plasma-splayed titanium stem: Bi-Metric (Biomet, Inc., Warsaw, IN) for 20 years [2]. In 2007 a new stem design: Echo Bi-Metric (Biomet, Inc., Warsaw, IN) was introduced with features including: a polished femoral neck with flat anterior and posterior 
geometry, reduced metaphyseal geometry option (Reduced Proximal Profile, RPP), a tapered and smooth stem tip, two horizontal offset options, and distal extension of the circumferential porous plasma spray around the proximal third of the implant (Figure 1).

Intraoperative and early postoperative periprosthetic fractures have emerged as a significant complication following THA. Intraoperative fracture is an issue to tackle as an uncemented stem has gained its popularity and there are few reports on stem design and intraoperative fracture. We hypothesized that changes in stem design would reduce the incidence of fracture and have an unknown effect on stem survivorship. The purpose of this study was to report the short- to mid-term results of Echo Bi-Metric stem comparing its predecessor.

\section{Methods}

From July 1986 to January 2014, we performed 3373 THAs with uncemented Bi-Metric and Echo Bi-Metric stems at our institution. Bi-Metric was utilized for 1606 hips from 1986 to 2008, Echo Bi-Metric with full proximal profile (Echo FPP) for 1374 hips and Echo Bi-Metric with reduced proximal profile (Echo RPP) for 393 hips from 2007 to 2014. Of these, we reviewed the eligible records of 1497 Bi-Metric stems in 1280 patients, 1277 Echo FPP stems in 1104 patients, 366 Echo RPP stems in 317 patients (Table 1). There was a cemented stem option in the Bi-Metric and we used it for Dorr type C, osteoporotic femur. But there was no cemented option with the Echo FPP or RPP. The use of these stems was surgeon selection based on subjective assessment of bone quality and endosteal geometry, offset, and the goal of restoration of the hip center of rotation. Final prosthetic selection was solely based on surgeon preference for a given case. In general RPP stems were selected for Dorr B and C type endosteal geometries to accommodate the relative mismatch of metaphyseal and proximal diaphyseal geometries. The average age of our patients at the surgery was $62.4,64.8$ and 63.2 years respectively. The average follow-up period was $7.6[0,26], 1.6[0,6.5]$ and $1.3[0,5.0]$ years respectively. In all cohorts, osteoarthritis was the most common preoperative diagnosis in $88.8 \%, 95.0 \%, 94.0 \%$ followed by avascular necrosis in $8.7 \%, 2.7 \%, 2.7 \%$ and rheumatoid arthritis in $1.3 \%, 0.6 \%$ and $2.5 \%$, respectively. Kaplan-Meier analysis of the survival of the femoral component was performed for all three cohorts. This analysis was used to compare hips with an intraoperative femoral fracture against those without. Logistic regression was used to analyze differences in intraoperative fracture rates between the three cohorts. Other variables included were gender, age, BMI, approach, and diagnosis. Significance level was set at $\mathrm{p}<0.05$.

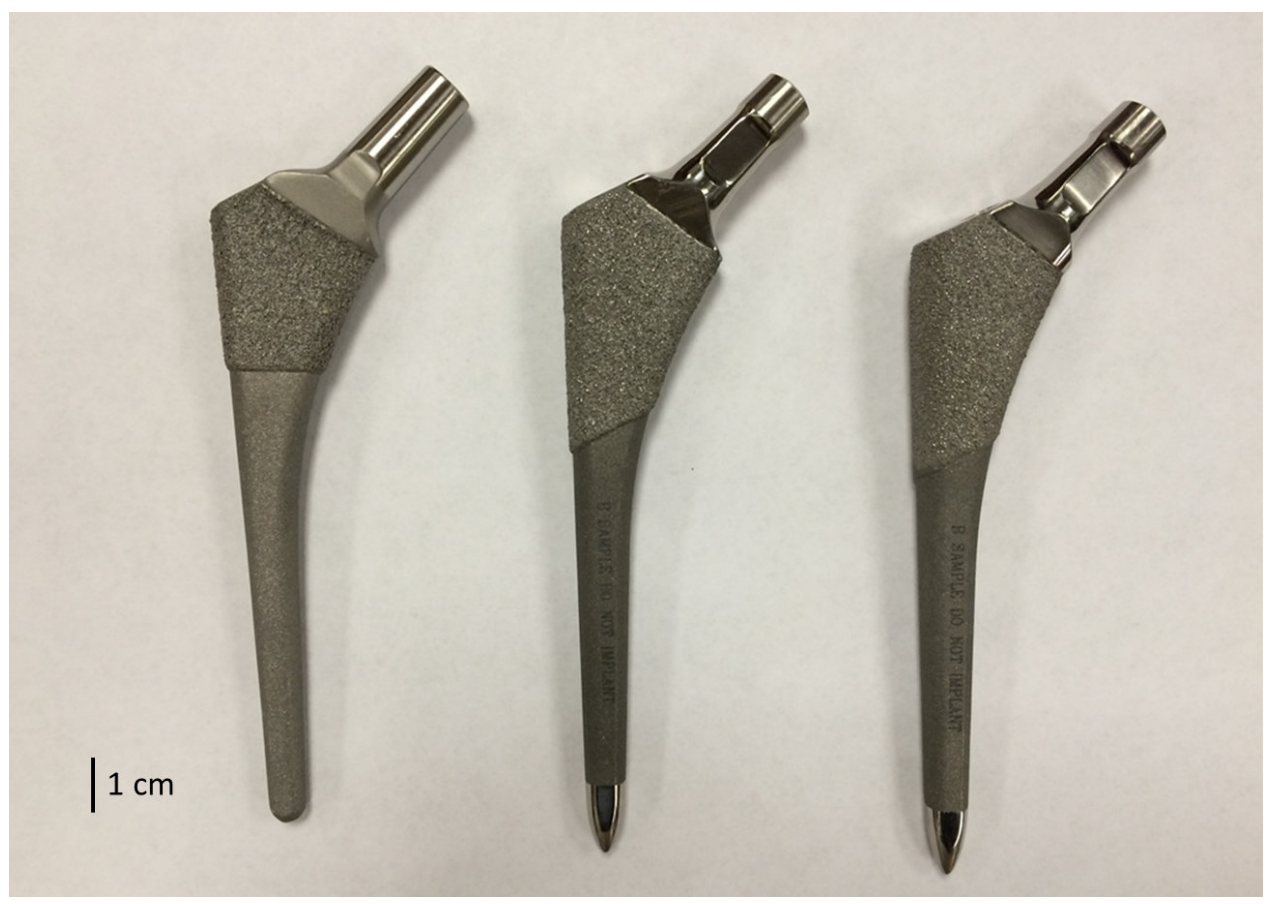

Figure 1. From left to right, Bi-Metric, Echo Bi-Metric full proximal profile (FPP), Echo Bi-Metric reduced proximal profile (RPP). Scale bar: $1 \mathrm{~cm}$. 
Table 1. Patients demographics.

\begin{tabular}{rcccc}
\hline & & Bi-Metric & Echo FPP & Echo RPP \\
\hline \multirow{2}{*}{ Hips } & Total Count & 1497 & 1277 & 366 \\
Female/Male & $633 / 864$ & $560 / 717$ & $325 / 41$ \\
Age [range] (yrs.) & $62.4[23,93]$ & $64.8[26,93]$ & $63.2[21,95]$ \\
Time of surgery & $1986-2008$ & $2007-2014$ & $2007-2014$ \\
F/u Period (yrs.) & $7.9[0,26.0]$ & $1.6[0,6.5]$ & $1.3[0,5.0]$ \\
BMI (kg/m $\left.{ }^{2}\right)$ & $30.0[16.7,58.1]$ & $31.2[18.6,60.3]$ & $31.1[16.1,54.1]$ \\
Approach & $549(36.7 \%)$ & $433(33.9 \%)$ & $261(71.3 \%)$ \\
& Anterolateral & $799(53.4 \%)$ & $835(65.4 \%)$ & $104(28.4 \%)$ \\
Posterior & $1329(88.8 \%)$ & $1213(95.0 \%)$ & $344(94.0 \%)$ \\
Diagnosis & $19(1.3 \%)$ & $8(0.6 \%)$ & $9(2.5 \%)$ \\
& OA & $130(8.7 \%)$ & $35(2.7 \%)$ & $10(2.7 \%)$ \\
\end{tabular}

Echo FPP: Echo full proximal profile; Echo RPP: Echo reduced proximal profile; F/u: Follow-up; OA: Osteoarthritis; RA: Rheumatoid arthritis; AVN: Avascular necrosis.

\section{Results}

There were 82 (5.48\%) intraoperative fractures in the Bi-Metric, 18 (1.41\%) fractures in the Echo Bi-Metric FPP, and $5(1.37 \%)$ fractures in the Echo Bi-Metric RPP. All the fractures experienced in this study occurred in the proximal part of the femur as classified as Vancouver type A. These fractures were mostly incomplete fractures, or cracks. All were recognized intraoperatively and were treated with Luque wire fixation to achieve vertical and rotational stability as tested immediately after wire placement and final implant insertion. The number of wires used varied from 1 to 2. Bi-Metric stems have 4.0 times the odds of intraoperative fracture compared to the Echo FPP stems. Bi-Metric stems have 6.0 times the odds of intraoperative fracture compared to the Echo RPP stems. There is no statistical difference between Echo FPP and RPP stems. In the Bi-Metric cohort, there were 60 fractures in 549 anterolateral approached hips and 22 fractures in 799 posterior approached hips. In the Echo Bi-Metric FPP cohort, there were 7 fractures in 433 anterolateral approached hips and 11 fractures in 835 posterior approached hips. In the Echo Bi-Metric RPP group, there were 3 fractures in 261 anterolateral approached hips and 2 fractures in 104 posterior approached hips.

Anterior approach had 3.1 times the odds of intraoperative fractures to posterior approach. Patients under 58 years old had 1.6 times the odds of intraoperative fractures to patients over 58 years old. The average age at the surgery was 62.4, 64.8 and 63.2 years with Bi-Metric, Echo FPP and Echo RPP respectively. Regression analysis revealed there was no significant difference of age among all the each cohorts.

There was no stem revision so far in all the Bi-Metric, Echo FPP and Echo RPP stems. Stem survivorship with aseptic loosening as an endpoint was also $100 \%$ in all cohorts at the final follow-up.

\section{Discussion}

Uncemented stems have gained popularity over the past 20 years. They have improved second decade stem survivorship compared to cemented stems; however, intraoperative fracture rates have increased [1]-[6]. The intraoperative fracture has been reported to have relevance to anterolateral surgical approach [1], female gender [6], diagnoses other than osteoarthritis of the hip [7], certain instrumentation [6] [8], and stem design [8]-[10]. The current study also demonstrated that stem design reduced the intraoperative fracture rate.

Recently, several reports demonstrated that stem design reduced proximal femoral fractures in primary [8] [9] and revision [10] surgery. Berend et al. reported that the fracture rate using a flat, wedge, tapered design is lower than that associated with previously published designs [8]. Molli et al. demonstrated the shorter stem decreased the intraoperative fractures and intraoperative complications [9]. Richards et al. experienced that using tapered, fluted, modular titanium stems instead of cylindrical, nonmodular cobalt chrome stems reduced intraoperative 
fractures and improved clinical outcomes in revision hip arthroplasty [10]. In this study, using similar proximal stabilizing concepts with the same porous coating but newly designed stems reduced the fracture rate, though a reduced proximal profile (RPP) does not significantly reduce the fracture rate comparing full proximal profile (FPP).

Expected mismatch between proximal femoral geometry and the femoral component are considered as a potential risk [1]. Diagnoses other than osteoarthritis such as developmental dysplasia of the hip, Perthes disease, and slipped capital femoral epiphysis may lead to altered proximal femoral geometry and result in this mismatch [8]. The Echo Bi-Metric has options to reduce proximal profile of the femur for a patient with a small femoral canal or Dorr Type C femur. Achieving the appropriate fit reduces the future risk of subsidence or shortening of the leg [11]. Our current results did not show significant difference among the various diagnoses. However, our demographics weighed too much on osteoarthritis in each cohort, there may have been a risk if diagnoses other than osteoarthritis had more number.

Previously, we reported risk factors of proximal femoral fracture in uncemented and cemented total hip arthroplasty [1]. We demonstrated that uncemented stem, anterolateral approach and female gender were risk factors. The current study supported the risk of anterolateral approach with the regression analysis with the Bi-Metric. But new designed stem; Echo Bi-Metric FPP and RPP did not show significant difference between anterolateral approach and posterior approach. We are not exactly sure what contributed to lowering the fracture rate in the anterolateral approach with the Echo Bi-Metric, but the change of instrumentation design could be a candidate as Berend et al. reported [9]. The Echo Bi-Metric has a developed calcar mill and options for full and half toothed broaches.

Age was a significant risk in the previous report but it was more likely related to the uncemented stem [1]. In the current study, patients younger than 58 years old had 1.6 times higher an odds of intraoperative fractures than patients older than 58 years old. Franklin et al reported female sex and increased age have been suggested to be independent risk factors but these factors may be confounded by osteoporosis [12]. Schwartz et al. experienced the population of patients older than 60 years old are larger in intraoperative fracture group than in the non-fracture group using uncemented total hip arthroplasty, but they found no significant difference [6]. An increase of intraoperative fractures is reported with the introduction of uncemented stems and this is often a consequence of the effort to obtain a sufficient press-fit to gain initial stem stability [13]. However, no other report has demonstrated a younger age has a higher risk. We surmise this is due to relatively solid cortical bone of the femur in younger patients and we have high population of OA patients and low population of younger RA patients, who are supposed to be osteopenia.

There are several limitations in this study. First, it was not a prospective study and each of 3 cohorts was not performed at the same period. Second, the committed surgeons were not exactly the same as it may have affected surgical approaches. Finally, the patients' demographics are not matched so the population of RPP resulted in mostly female patients. The indication of the surgery was not exactly matched.

\section{Conclusion}

In conclusion, stem design was one of the important factors which reduced intraoperative fractures in our uncemented total hip arthroplasties. We found that the renewed stem reduced intraoperative fractures and showed $100 \%$ survivorship. While it is still early to evaluate the Echo FPP and the Echo RPP long-term, we are encouraged by these outcomes and use this stem on a regular basis.

\section{Conflict of Interest Statement}

None of the authors has received or will receive benefits for personal or professional use from a commercial party directly or indirectly related to the subject of this article.

\section{Acknowledgements}

We would like to thank Matthew Brunsman and Kenneth Davis for their hard work in the data collection and statistics.

\section{References}

[1] Berend, M.E., Smith, A., Meding, J.B., et al. (2001) Long-Term Outcome and Risk Factors of Proximal Femoral Frac- 
ture in Uncemented and Cemented Total Hip Arthroplasty in 2551 Hips. Journal of Arthroplasty, 21, 53-59. http://dx.doi.org/10.1016/j.arth.2006.05.014

[2] Meding, J.B., Keating, E.M., Ritter, M.A., et al. (2004) Minimum Ten-Year Follow-Up of a Straight-Stemmed, Plasma-Sprayed, Titanium-Alloy, Uncemented Femoral Component in Primary Total Hip Arthroplasty. Journal of Bone and Joint Surgery (American), 86, 92-97.

[3] Lombardi Jr., A.V., Berend, K.R., Mallory, T.H., et al. (2009) Survivorship of 2000 Tapered Titanium Porous PlasmaSprayed Femoral Components. Clinical Orthopedics Relatated Research, 467, 146-154. http://dx.doi.org/10.1007/s11999-008-0568-X

[4] Marshall, A.D., Mokris, J.G., Reitman, R.D., et al. (2004) Cementless Titanium Tapered-Wedge Femoral Stem: 10- to 15-Year Follow-Up. Journal of Arthroplasty, 19, 546-552. http://dx.doi.org/10.1016/j.arth.2004.01.006

[5] McLaughlin, J.R. and Lee, K.R. (2010) Uncemented Total Hip Arthroplasty with a Tapered Femoral Component: A 22- to 26-Year Follow-Up Study. Orthopedics, 33, 639. http://dx.doi.org/10.3928/01477447-20100722-40

[6] Schwartz Jr., J.T., Mayer, J.G. and Engh, C.A. (1989) Femoral Fracture during Non-Cemented Total Hip Arthroplasty. Journal of Bone and Joint Surgery (American), 71, 1135-1142.

[7] Berend, K.R., Lombardi Jr., A.V., Mallory, T.H., et al. (2004) Cerclage Wires or Cables for the Management of Intraoperative Fracture Associated with a Cementless, Tapered Femoral Prosthesis: Results at 2 to 16 Years. Journal of Arthroplasty, 19, 17-21. http://dx.doi.org/10.1016/j.arth.2004.06.008

[8] Berend, K.R. and Lombardi Jr., A.V. (2010) Intraoperative Femur Fracture Is Associated with Stem and Instrument Design in Primary Total Hip Arthroplasty. Clinical Orthopedics Related Research, 468, 2377-2381. http://dx.doi.org/10.1007/s11999-010-1314-8

[9] Molli, R.G., Lombardi Jr., A.V., Berend, K.R., et al. (2012) A Short Tapered Stem Reduces Intraoperative Complications in Primary Total Hip Arthroplasty. Clinical Orthopedics Related Research, 470, 450-461. http://dx.doi.org/10.1007/s11999-011-2068-7

[10] Richards, C.J., Duncan, C.P., Masri, B.A., et al. (2010) Femoral Revision Hip Arthroplasty: A Comparison of Two Stem Designs. Clinical Orthopedics Related Research, 468, 491-496. http://dx.doi.org/10.1007/s11999-009-1145-7

[11] Andrews, S., Bentall, S. and Atkinson, D. (2006) Early Subsidence of Accolade Uncemented Total Joint Replacement. Journal of Bone Joint Surgery (British), 88, 321.

[12] Franklin, J. and Malchau, H. (2007) Risk Factors for Periprosthetic Femoral Fracture. Injury, 38, 655-660. http://dx.doi.org/10.1016/j.injury.2007.02.049

[13] Lindahl, H. (2007) Epidemiology of Periprosthetic Femur Fracture around a Total Hip Arthroplasty. Injury, 38, 651654. http://dx.doi.org/10.1016/j.injury.2007.02.048 